\title{
Construcción del Índice de Riesgo para determinar la Sostenibilidad del Servicio de Agua y Saneamiento (IRSSAS) en distritos rurales de Costa Rica atendidos por ASADAs
}

\section{Construction of Risk-Index for assessment of water and sanitation sector (IRSSAS) in Costa Rica}

\author{
Macario Pino-Gómez¹, Silvia M. Soto-Córdoba², \\ Lilliana Gaviria-Montoya ${ }^{3}$
}

Fecha de recepción: 1 de junio de 2020

Fecha de aprobación: 29 de setiembre de 2020

Pino-Gómez, M; Soto-Córdoba, S.M; Gaviria-Montoya, L. Construcción del Índice de Riesgo para determinar la Sostenibilidad del Servicio de Agua y Saneamiento (IRSSAS) en distritos rurales de Costa Rica atendidos por ASADAs. Tecnología en Marcha. Vol. 34-3 Julio-Setiembre 2021. Pág 156-176.

doi https://doi.org/10.18845/tm.v34i3.5198

1 Centro de investigación y Protección Ambiental (CIPA), School of Enviromental Engineering , Instituto Tecnológico de Costa Rica, Cartago, Costa Rica. Correo electrónico: mpino@tec.ac.cr (iD https://orcid.org/0000-0001-8446-4723

2 Centro de investigación y Protección Ambiental (CIPA), School of Chemistry, Instituto Tecnológico de Costa Rica, Apartado 159-7050, Cartago, Costa Rica. Correo electrónico: ssoto@tec.ac.cr (iD https://orcid.org/0000-0002-3550-1505

3 Centro de investigación y Protección Ambiental (CIPA), School of Chemistry, Instituto Tecnológico de Costa Rica, Apartado 159-7050, Cartago, Costa Rica. Correo electrónico: Igaviria@tec.ac.cr 


\title{
Palabras clave
}

Agua potable; cambio climático; gobernanza del agua; índice riesgo; acueductos rurales.

\section{Resumen}

Las amenazas del recurso hídrico son multifactoriales, incluyen la degradación y contaminación de acuíferos y suelos, gestión de los acueductos, aspectos financieros, educativos y características propias del territorio. La presión de uso del agua y la variabilidad en su oferta debido al cambio de clima, justifican la urgente necesidad de contar con herramientas predictivas del riesgo. Costa Rica carece de un índice que prediga el riesgo en la prestación sostenible de los servicios de agua potable y saneamiento. Por estas razones se desarrolló y se estimó a través de una investigación de varios años, lo que se denomina el IRSSAS, Índice de Riesgo en la Sostenibilidad de la prestación del Servicio de agua potable y Saneamiento. Dicho índice se construyó considerando la Gestión del Recurso Hídrico (GRH), el Saneamiento Ambiental (SA), la Educación Ambiental (EA), el Índice de Desarrollo Socioeconómico (IDS) y el impacto de los Eventos Naturales (EN). En total se diseñaron, validaron y calcularon, 30 indicadores que componen el índice. Los indicadores se evaluaron considerando su aporte a la vulnerabilidad, mitigación, pertinencia, funcionalidad, disponibilidad, confiabilidad y utilidad. El índice se aplicó en una muestra de 97 acueductos rurales ubicados en las provincias de Puntarenas, Guanacaste y Cartago. Los resultados de la evaluación del riesgo mostraron que el $81 \%$ de esos acueductos presentaban niveles de riesgo superior al intermedio, lo cual evidencia la poca sustentabilidad en la prestación del servicio de agua y saneamiento por parte de dichos entes operadores. Este índice podrá ser utilizado para evaluar el riesgo y tomar acciones correctivas y preventivas colaborando así con el desarrollo sostenible del país.

\section{Keywords}

Drinking water; climate change; water governance; risk index; rural water suppliers.

\begin{abstract}
The threats to water resources are multifactorial, including the degradation and contamination of aquifers and soils, management of aqueducts, financial aspects, education and the characteristics of the territory. The pressure of water use and the variability in its supply due to climate change, justify the urgent need for risk prediction tools. Costa Rica lacks an index that predicts risk in the sustainable provision of drinking water and sanitation services. For these reasons, the research study developed during 2014 to 2019 to estimate an index named Risk Index in the Sustainability of Drinking Water and Sanitation Service Provision (IRSSAS). This index was constructed by considering Water Resource Management (WRM), Environmental Sanitation (ES), Environmental Education (EA), Socioeconomic Development Index (SDI) and the impact of Natural Events $(\mathrm{NE})$. A total of 30 indicators were designed, validated and calculated to make up the index. The indicators were evaluated considering their contribution to vulnerability, mitigation, relevance, functionality, availability, reliability and usefulness. The index was applied to a sample of 97 rural aqueducts located in the provinces of Puntarenas, Guanacaste and Cartago. The results of the risk assessment showed that $81 \%$ of these aqueducts presented higher than intermediate risk levels, which shows the lack of sustainability in the provision of water and sanitation services by these operators. This index can be used to evaluate risk and take corrective and preventive actions, thus collaborating with the country's sustainable development.
\end{abstract}




\section{Lista de acrónimos}

ASADA: Asociaciones Administradoras de Sistemas de Acueductos y Alcantarillados Comunales ARESEP: Autoridad reguladora de los servicios públicos.

AyA: Acueductos y alcantarillados

IRSSAS: Índice de Riesgo en la Sostenibilidad de la prestación del Servicio de agua potable y Saneamiento

MINAE: Ministerio de Ambiente y Energía

CGR: Contraloría General de la República

MIDEPLAN: Ministerio de Planificación Nacional y Política Económica

MinSalud: Ministerio de Salud

INEC: Instituto Nacional de Estadística y Censos

IMN: Instituto Meteorológico Nacional

SERSA: Sistema Estandarizado de Regulación de la Salud

EA: Educación Ambiental

SA: Saneamiento Ambiental

GRH:Gestiónde Recurso Hídrico

EN: Eventos Naturales

IDS: Índice de Desarrollo Social

IGN: Instituto Geográfico Nacional

\section{Introducción}

En las zonas rurales de Costa Rica, la distribución del agua es brindada por Asociaciones Administradoras de Sistemas de Acueductos y Alcantarillados Comunales (ASADAs), [1] [2]. Las ASADAs atienden un 29 \% de la población del país, siendo que el 73,2 \% brindan el agua en comunidades de menos de 300 abonados [3]. En el cuadro 1 se muestra la distribución de ASADAs en el país y la cantidad de abonados atendidos.

Cuadro 1. Distribución de las ASADAs según provincia y cantidad de abonados y población atendida en Costa Rica.

\begin{tabular}{|c|c|c|c|c|c|c|c|c|}
\hline & \multicolumn{7}{|c|}{ Provincias } & \\
\hline Aspecto & San José & Heredia & Cartago & Alajuela & Puntarenas & Guanacaste & Limón & Total País \\
\hline \#abonados & 53206 & 27204 & 46997 & 151238 & 55312 & 47455 & 34771 & 416183 \\
\hline \# ASADAs & 225 & 41 & 131 & 323 & 181 & 276 & 98 & 1275 \\
\hline $\begin{array}{c}\text { \#abonados } \\
\text { promedio/ } \\
\text { ASADA }\end{array}$ & 236 & 664 & 359 & 468 & 306 & 172 & 355 & N.A. \\
\hline $\begin{array}{l}\text { Población } \\
\text { atendida* }\end{array}$ & 239427 & 12418 & 211487 & 680571 & 248904 & 213548 & 156470 & 1872824 \\
\hline
\end{tabular}

* El cálculo se realiza considerando un promedio de 4,5 habitantes por abonado.

Fuente: Elaboración propia con datos provenientes del sitio Web de Acueductos y Alcantarillados [4] 
Las ASADAs presentan deficiencias asociadas a la inadecuada gestión de las infraestructuras de captación, conducción y distribución [5], administrativas y financieras [6], deterioro o contaminación de sus fuentes primarias por actividades agrícolas-ganaderas y por el uso de sustancias químicas [7], ausencia, dificultades y falta de capacitación asociadas a los sistemas de desinfección [8], contaminación del agua con coliformes fecales [9], insuficientes tratamientos de las aguas residuales [10], daños a las infraestructuras derivadas de los eventos naturales como inundaciones por tormentas tropicales, huracanes o lluvias torrenciales [11], sismos [12], vulnerabilidad en las zonas de protección inmediata por brechas volcánicas [13] y deslizamientos derivados de las condiciones del terreno y lluvias [14].

La infraestructura del suministro de agua por medio de las ASADAs se ha comenzado a deteriorar y al menos, la mitad de los acueductos ya requieren reparaciones y rehabilitación, sin disponer de recursos para tal fin, los préstamos no son una opción viable, pues la mayoría de población de las zonas son pobres. La relación de las ASADAs con AyA es de subordinación, pero este último no cuenta con capacidad para promover y regular a las ASADAs, existe ambigüedad en el papel dual de rector y actor, lo que dificulta la gestión de estas [15].

Los instrumentos de control y monitoreo que se aplican a las ASADAs incluyen los denominados planes de seguridad del agua [16], formulario unificado [9], Sistema Estandarizado de Regulación de la Salud (SERSA) [17], herramienta de la gestión integral del riesgo (GIRA) [18], los cuales se enfocan en regulación, monitoreo y no brindan una retroalimentación a los funcionarios de las ASADAs, tampoco están concebidos para mitigar los efectos del cambio climático.

El cambio climático tendrá un impacto directo en la distribución del agua en todo el país, se prevén afectaciones por escases en las zonas de Guanacaste [19], donde se proyectan déficits del orden del $15 \%$, así mismo, se proyectan aumentos de precipitación en la zona del caribe, lluvias torrenciales acompañadas de periodos de sequias [20].

Los escenarios propuestos para mitigar el impacto del cambio climático asociados al recurso hídrico incluyen, la consolidación del sistema boscoso, el aumento de alcantarillados, la construcción de plantas de tratamiento de aguas residuales, el manejo adecuado de los residuos sólidos, una economía baja en emisiones, disminución del consumo de agua por habitante y el aumento en el IDS [21] [22].

A la fecha, la principal acción gubernamental ante el cambio climático consiste en el programa nacional de descarbonización, orientado principalmente a disminuir las emisiones de gases con efecto invernadero provenientes de los sectores de transporte, tratamiento de residuos sólidos y empresas [23].

En Costa Rica, el INEC realiza periódicamente estadísticas ambientales en seis componentes: Condiciones y calidad ambiental, recursos ambientales y su utilización, emisiones, residuos y desechos, eventos extremos y desastres, hábitat humano y salud ambiental y protección ambiental, gestión y participación/acción ciudadana [24]. El Ministerio de Ambiente y Energía también dispone del SINIA [25] [26], portal que incluye un anuario estadístico del Centro Nacional de Información Geo-ambiental (CENIGA), Estadísticas del estado del ambiente, indicadores ambientales de la Iniciativa Latinoamericana y Caribeña, estadísticas respecto a los objetivos del desarrollo sostenible y estadísticas del agua, estas últimas administradas por la dirección de agua [27]. Los sistemas nacionales de información se enfocan en atmósfera, recurso hídrico, tierra, residuos, biodiversidad, bosque, recurso marino y pesquería, energía, vulnerabilidad y asentamientos humanos y compromiso y protección ambiental, brindando más de 1748 datos en 300 variables [28]. Adicionalmente CONARE [29] publica anualmente el documento denominado Estado de la Nación, donde investigadores expertos analizan las estadísticas ambientales. El gobierno de la República también genera su propio informe del 
estado del ambiente [30] y hasta el Banco Central de Costa Rica (BCCR) tiene un sistema de contabilidad ambiental y económica (SCAE) para agua, bosques y energía con el objetivo de conocer la oferta y utilización de dichos recursos [31]. La Organización para la Cooperación y el Desarrollo Económico (OCDE) ha reconocido la calidad de los datos que se recopilan y las estadísticas nacionales en materia ambiental [32]

La capacidad de respuesta ante el cambio de clima asociada al sector hídrico requiere diversas acciones estructurales, dentro de las que se incluyen la construcción de infraestructura, planificación, políticas, acciones culturales, educación ambiental, financiamiento para proyectos, entre otros.

Con el fin de ejecutar acciones para contrarrestar los efectos del cambio en los patrones de Iluvia, resulta necesario conocer los factores de riesgo que afectarán la sustentabilidad del servicio de prestación del agua y la presión sobre los sistemas de saneamiento. Por tanto, se propone la construcción de un índice de sustentabilidad para el sector.

Los índices deben construirse de forma que provean suficientes bases para medir, pero a la vez, deben ser simples y fáciles de aplicar, de forma que no se conviertan en una acción compleja de realizar. Existen diversos tipos de indices de sustentabilidad aplicables a el manejo de aguas residuales [33], eficiencia del manejo de los recursos en los paises [34], uso del agua [35] [36], calidad del agua [37], entre otros, estos son complejos y requieren de información que no se recolecta en el país.

EI MIDEPLAN administra el Índice de Desarrollo Social que tiene como objetivo ordenar los distritos y cantones según su nivel de desarrollo social, y se calcula a partir de 14 índices socioeconómicos [38], así mismo, la Contraloría General de la Republica en el sistema integrado de información municipal (SIIM) administra un índice de gestión municipal donde uno de los componentes es la gestión de residuos sólidos [39], ambos tienen relación directa con la sustentabilidad en la prestación del servicio de agua y saneamiento. Los indicadores que ha desarrollado el país y en Latinoamérica, corresponden a indicadores ambientales que ocupan describir y mostrar las dinámicas ambientales, a la fecha no se disponen de indicadores de desarrollo sostenible, que muestren las dinámicas socioeconómicas - [40]

La construcción de los índices debe estar apegados a los objetivos de la medición, y en este caso, los investigadores, proponen la construcción de un índice cuantitativo para determinar el riesgo en la sostenibilidad de la prestación del servicio de agua potable y saneamiento, cuyo acrónimo es IRSSAS. El índice formulado hace uso de la información oficial disponible que actualmente se recolecta en forma rutinaria en el país; está formulado para ser aplicado en cada operador de agua y los resultados se grafican sobre mapas usando como unidad el distrito. EI IRSSAS se focaliza en el servicio prestado por entes operadores de agua y saneamiento. El mismo fue diseñado para ser accesible, aplicable, confiable, disponible, observable, relevante, representativo, válido, y verificable. EI IRSSAS es un indicador de impacto, cuyo fin es medir el riesgo de la sostenibilidad de la prestación del servicio de agua y saneamiento en las comunidades atendidas por ASADAs. La sustentabilidad en la dotación del agua potable en las zonas rurales, requiere la debida atención de aquellas variables que afectan los ecosistemas, tales como contaminación, extracción excesiva del agua, uso inadecuado del recurso y también, depende de la capacidad de las poblaciones para acceder al agua, mediante infraestructura apropiada de captura y conducción, así como, tratamientos de desinfección y controles de calidad del agua, todo lo anterior de vital relevancia ante el panorama del cambio climático y el crecimiento de la población. 


\section{Materiales y métodos}

Identificación de los objetivos del índice

Para construir el índice del riesgo en la prestación del servicio sostenible de agua y saneamiento, los investigadores plantearon que dicho índice, debía cumplir con los siguientes objetivos:

- Colaborar con los entes operadores de agua y saneamiento y los gobiernos locales, en los procesos de planificación y gestión ambiental.

- Ofrecer información sistematizada y comparativa del riesgo sostenible en la prestación del servicio de agua y saneamiento de los entes operadores.

- Servir de guía a las instituciones rectoras del sector agua y saneamiento a fin de priorizar la intervención en los acueductos que presenten mayor riesgo.

- Servir de referencia con información actualizada para consulta de las instituciones rectoras, ASADAs, Municipalidades y público en general.

- Contribuir con la planificación del recurso hídrico como estrategia de mitigación ante el cambio climático.

- Contribuir con información sistematizada para enfocar las inversiones en áreas estratégicas.

- Colaborar con los entes operadores en el cumplimiento de la legislación ambiental nacional.

- Ofrecer herramientas a las Municipalidades para la gestión territorial de los distritos.

Proceso de recopilación de la información

El levantamiento de la información referente a la cantidad de ASADAs, ubicación, tamaño, contactos, la forma en que gestionan su administración y operación, se realizó durante los años 2014 hasta el 2017, para tal fin, se utilizaron las siguientes fuentes secundarias:

- Registros Administrativos (MINAE [26], MIDEPLAN [41], MinSalud [42], IFAM [43], CGR [44], IMN [19], AyA [4] [1] [45]), CNE [46]

- Censos de población, vivienda, distrito y cantones (INEC) [24] [47]

- Revisión de información técnica recopilada por academia y ONGs

- Revisión de artículos científicos que detallan los diferentes índices que se utilizan a nivel internacional. [33] [35] [48]

- Mapas oficiales provenientes del Instituto Geográfico Nacional (SNIT) [49]

Oficiales de la Regional de Salud del Ministerio de Salud en Cartago facilitaron una base de datos con información actualizada de las ASADAs ubicadas en la provincia, número de abonados, sistemas de desinfección, contactos y otras particularidades. Con esta base de datos se procedió en el año 2014, a seleccionar una muestra representativa de estudio para establecer la línea base de los indicadores iniciales [50].

Posteriormente, durante los años 2015 al 2016, se levantó información primaria a partir de talleres de trabajo con los encargados y fontaneros de las ASADAs seleccionadas, visitando las nacientes, sistemas de desinfección y oficinas centrales [51]. Se aplicaron los documentos del sistema estandarizado de regulación de la salud SERSA [52] y el formulario unificado de información sobre organizaciones comunales prestadoras de servicios de agua potable y saneamiento del AyA [53]. Con esta información se identificaron las principales vulnerabilidades asociadas a los acueductos comunales. Esto permitió la integración de datos y microdatos en una estructura que dio origen a la propuesta preliminar de matriz de riesgo. 


\section{Construcción de la matriz de riesgo}

La muestra de estudio correspondió a 97 Asadas, que representan más de un 7,6 \% del total de ASADAs del país.

La matriz preliminar es una serie de datos estadísticos que requieren validarse y organizarse, con el objetivo que el conjunto de datos sea robusto, descriptor y fácil de completar. Por esta razón, la matriz preliminar se optimizó mediante el uso de herramientas estadísticas con el fin de eliminar aquellos indicadores no predictoras o cuyo impacto fuera poco o insignificante en el resultado final de la ecuación del riesgo [34], el detalle de las herramientas estadísticas esta fuera del objetivo de este artículo.

Cada indicador de la matriz fue sometida al análisis indicado en la figura 1, donde se evaluó su capacidad de predecir el riesgo considerando los componentes de amenaza, vulnerabilidad, mitigación, relevancia, funcionalidad, datos, rigurosidad y utilidad, según lo descrito en las diversas metodologías para evaluar amenazas y riesgos [40], [54], [55]. Los indicadores que pasaron la prueba de pertinencia fueron agregadas a la matriz final.

El proceso realizado se sintetiza en la figura 1. El resultado del proceso generó los indicadores finales.

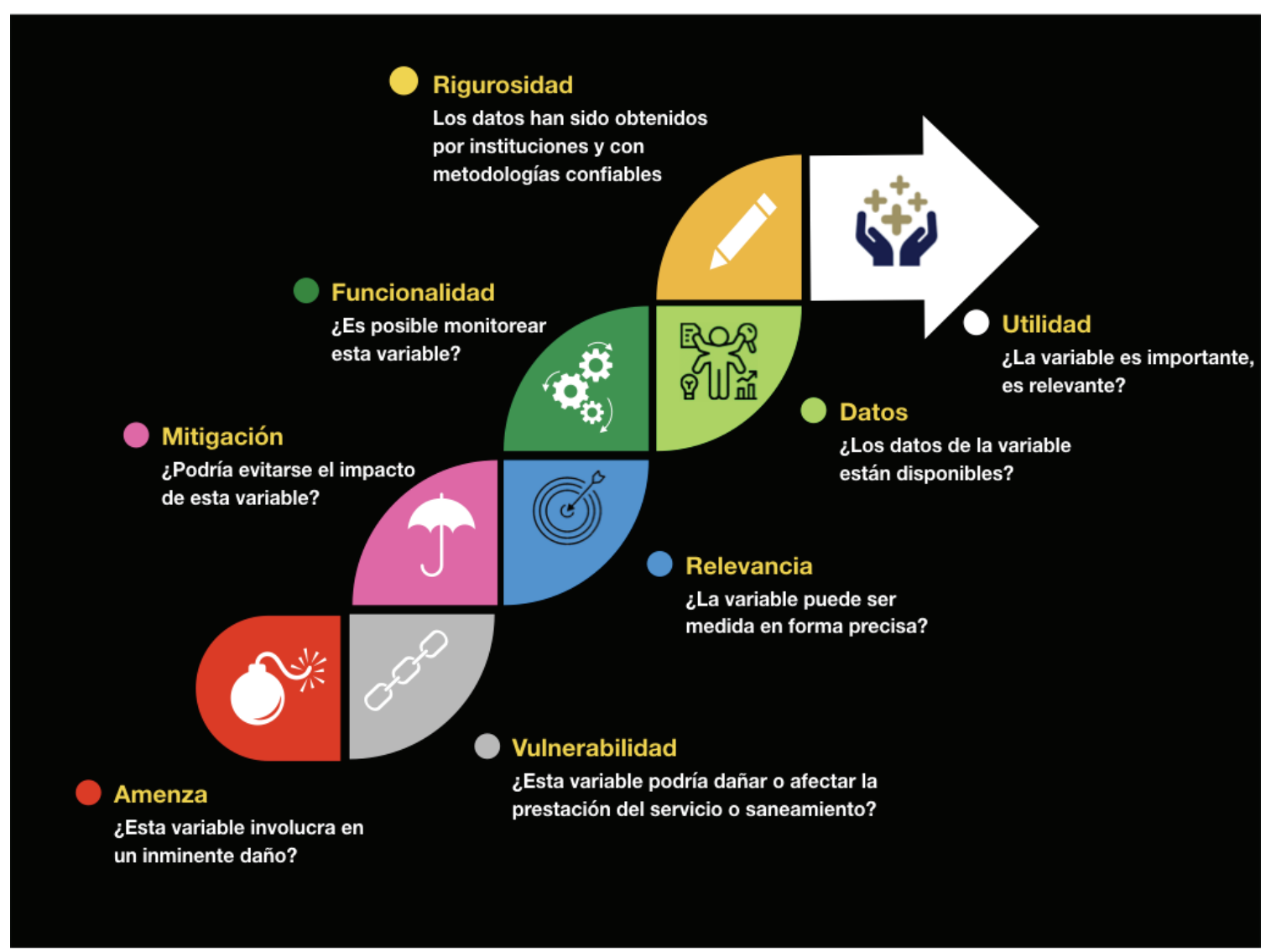

Figura 1. Infograma del análisis a cada una de los indicadores.

La selección de componentes y subcomponentes y los pesos, se realizó mediante cuestionarios a expertos institucionales, personal de las ASADAs y consulta de académicos, utilizando para ello, una adaptación de la metodología DELPHI que se describe en la figura 2. 


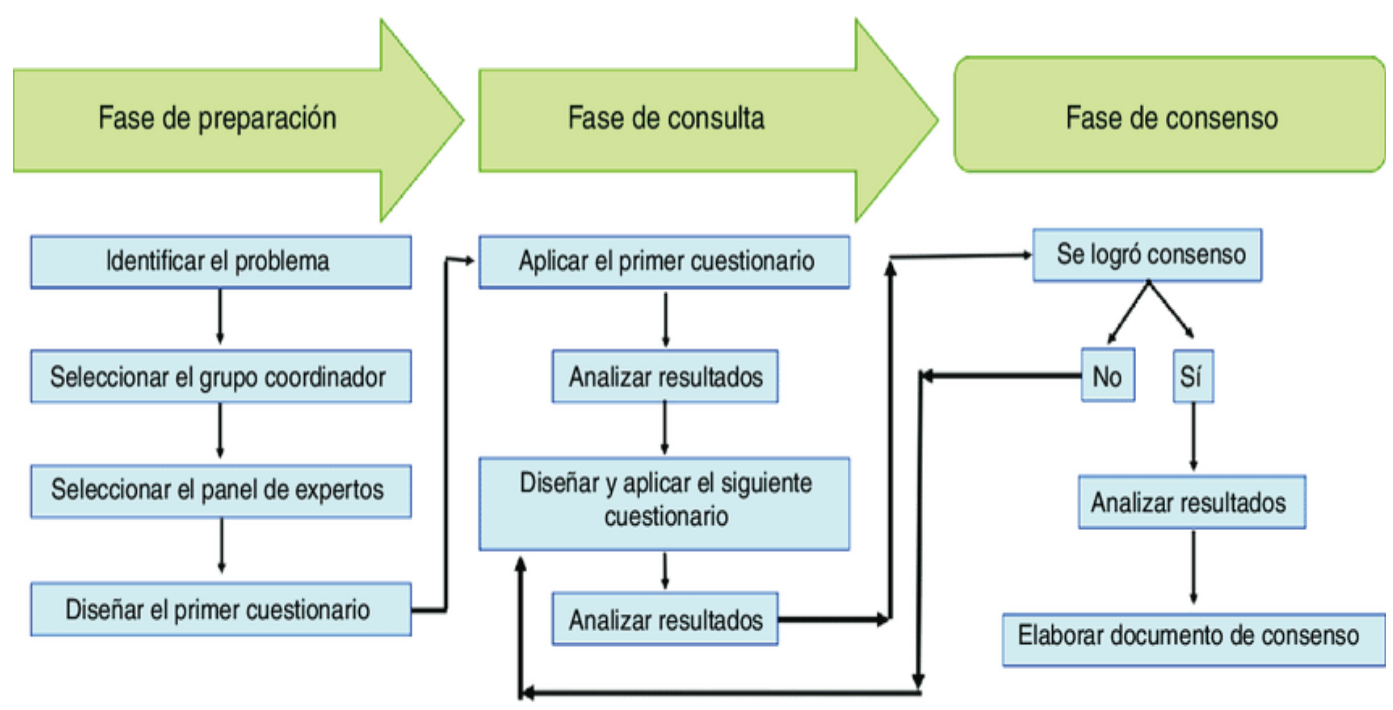

Figura 2. Pasos seguidos para selección de componentes y subcomponentes, adaptación de la metodología Delphi. [56]

En los cuestionarios aplicados se consideraron los siguientes aspectos:

- El impacto de la contaminación en el recurso hídrico

- La gestión del recurso hídrico

- Las inversiones en infraestructura (captación, conducción, tratamiento, almacenamiento, desinfección y distribución)

- Las condiciones socioeconómicas como predictores para responder al desarrollo sostenible.

- El aporte de las poblaciones concientizadas ambientalmente.

- Los riesgos asociados a la vulnerabilidad del territorio.

Para realizar los cálculos sobre esta nueva matriz, se utilizaron los datos suministrados por AyA, que correspondían a una muestra de 76 ASADAs ubicadas en las provincias de Guanacaste y Puntarenas. La construcción de la ecuación y de las escalas de riesgo para cada indicador de cada componente y subcomponente, se definieron con el aporte de un profesional en matemática aplicada, la forma en que se realizó este proceso se publicará en otro artículo. Una vez optimizada la ecuación se validó mediante comparación de resultados con datos de campo, para lo cual se realizaron visitas a 21 ASADAs en la provincia de Cartago y con esto se revisó nuevamente los resultados, para ajustar las escalas y optimizar los ajustes de la ecuación.

Una vez concluida la etapa de validación se procedió a establecer las fichas descriptivas (metadatos) para cada indicador, en las fichas se incorporaron los datos necesarios para normalizar la información, a saber: nombre del indicador, fuente, unidades, codificación, frecuencia de medición, ecuación, límite superior e inferior.

En la figura 3 se muestra un infograma que resume la metodología para la construcción del Índice. 


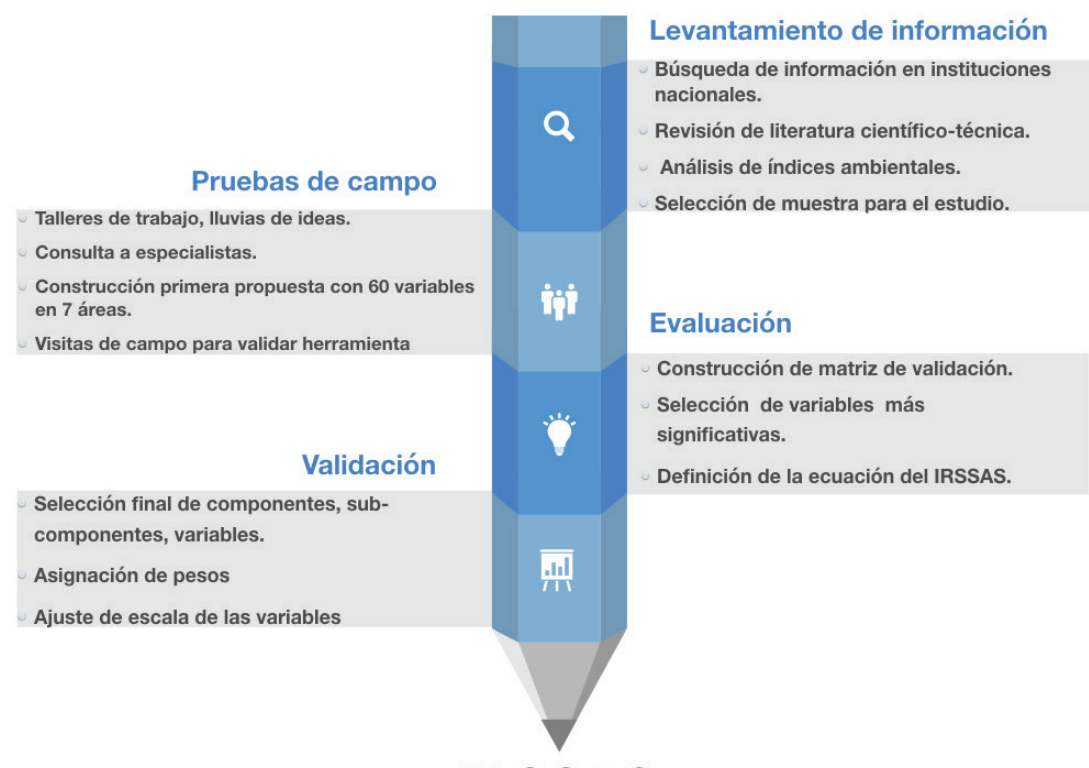

\section{IRSSAS}

Figura 3. Infograma de la conceptualización del índice IRSSAS.

El re-escalamiento se realizó empleando los valores entre los máximos y mínimos como indica [55].

\section{Construcción de mapas}

Para automatizar el cálculo del índice se diseñó un software con la ayuda de estudiantes de la carrera de Ingeniería en Computación del ITCR. El detalle de la construcción del software esta fuera de los objetivos de esta publicación. En el software se programó la base de datos de los resultados con los indicadores validados en la muestra de estudio. Se utilizaron los mapas que pone a disposición el IGN para mostrar la ubicación de las ASADAs así como el valor del IRSSAS obtenido en cada caso. Con el fin de visualizar el riesgo en el mapa, se utilizó la unidad geográfica del distrito. En los distritos con solo una ASADA, se les asignó un valor de IRSSAS idéntico al valor de la ASADA. En los distritos que contaban con más de una ASADA, para calcular el valor del IRSSAS se calculó el promedio ponderado del valor de los IRSSAS de cada ASADA y su población servida.

\section{Resultados}

\section{Determinación de vulnerabilidades}

Durante los años 2014 hasta el 2016 se recolectó información primaria proveniente de 21 ASADAs de la provincia de Cartago: Cooperosales [57], Quebradilla [58], Guatuso [59], Higuito [60], San Isidro [61], Macho Gaff [62], Cot [63], Paso Ancho y Boquerón [64], Potrero Cerrado [65], San Pablo [66], Santa Rosa [67], El Yas [68], Palomo [69], Río Macho [70], San Jerónimo [71], Santiago [72], San Vicente [73],Alto Varas [74], Jicotea [75], Santa Cristina [76] y Santa Cruz [77], en el cuadro 2 se resumen las principales vulnerabilidades encontradas en las ASADAS de la muestra de estudio. 
Cuadro 2. Resumen de vulnerabilidades de la muestra de estudio de ASADAs.

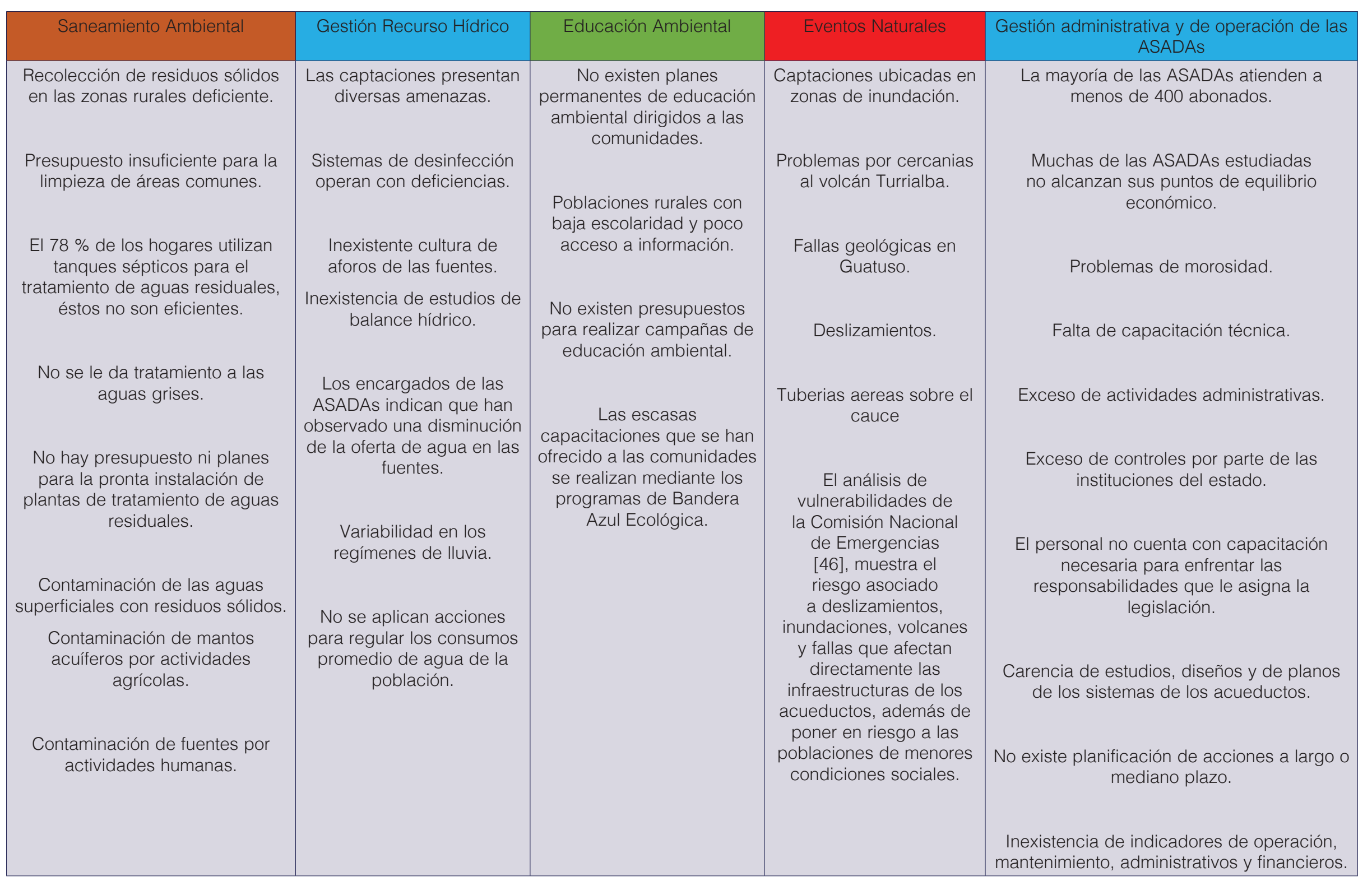


Matriz de riesgo

Se construyó la primera matriz de riesgo compuesta por 60 indicadores distribuidos en 7 componentes que componían el índice IRSSAS.

Luego del análisis de pertinencia de los indicadores según la metodología mostrada en la figura 1, éstos se redujeron hasta 30 indicadores que se enlistan en el cuadro 3. Se definieron cinco componentes: Recurso Hídrico, Saneamiento Ambiental, Educación Ambiental, Desarrollo Económico y Social de la región y Eventos Naturales asociados al territorio. Adicionalmente, éstos a su vez, se subdividieron en ocho subcomponentes: Gestión de Aguas Residuales, Gestión Integral de Residuos Sólidos, Calidad del agua potable, Gestión Administrativa financiera, índice de desarrollo social, educación ambiental y eventos naturales, los mismos se detallan en la figura 4.

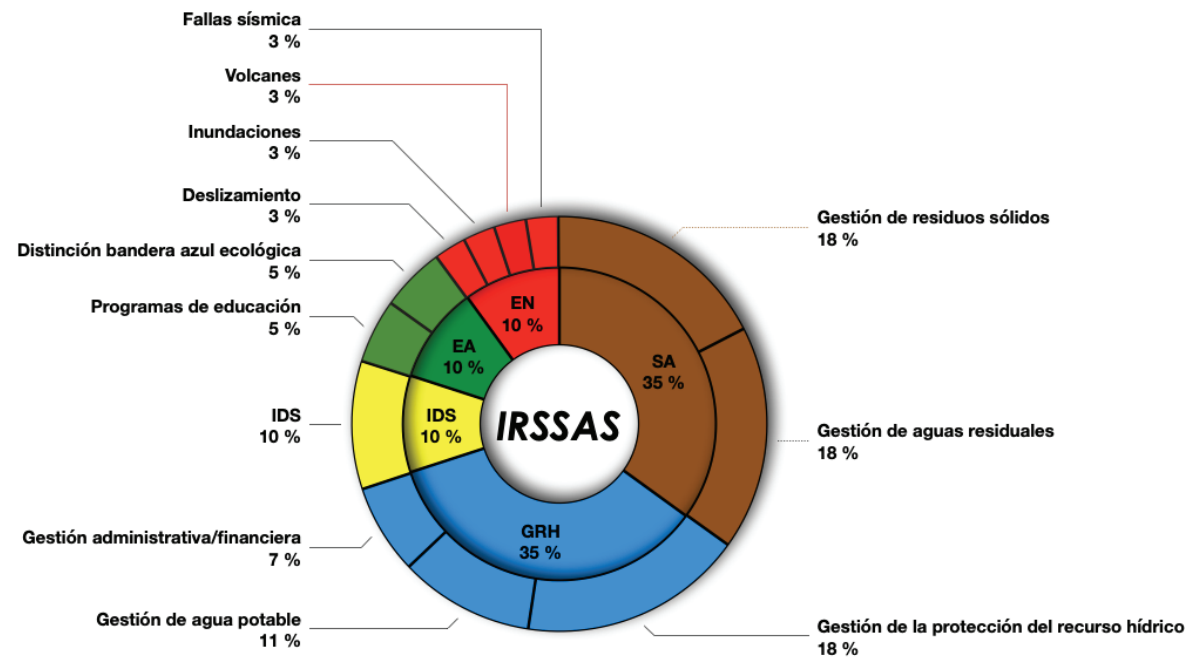

Figura 4. Composición del índice IRSSAS. SA: Saneamiento Ambiental, GRH: Gestión del recurso hídrico, IDS: Índice de Desarrollo Social, EA: Educación Ambiental, EN: Eventos naturales.

Con base en lo anterior se construyó la ecuación 1, esta muestra el análisis lógico de la composición del IRSSAS, donde se asignan pesos a cada uno de los componentes:

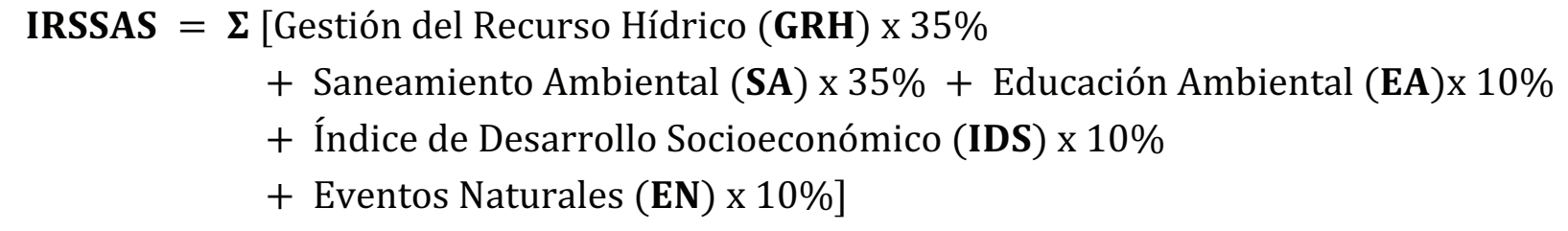

(Ecuación 1)

En el cuadro 3 se muestran indicadores seleccionados a partir de las cuales se calcula el IRSSAS, estos responden a los objetivos planteados. 
Cuadro 3. Matriz utilizada para el cálculo del IRSSAS.

\begin{tabular}{|c|c|c|}
\hline \multirow{5}{*}{ Componente } & Subcomponente & Indicador \\
\hline & \multirow{4}{*}{ Gestión AR } & $\begin{array}{l}\text { ¿Existen sistemas de tratamiento individual de aguas negras en la } \\
\text { comunidad? } \\
\text { ¿Existe presencia de aguas grises en los caños de recolección de agua } \\
\text { Iluvia? }\end{array}$ \\
\hline & & La población cuenta con alcantarillado pluvial? \\
\hline & & ¿La población tiene planta de tratamiento de aguas residuales? \\
\hline & & Porcentaje (\%) de liquidez para invertir en mejoras del acueducto \\
\hline \multirow[t]{5}{*}{ Saneamiento } & \multirow{5}{*}{ Gestión de RS } & \% Cobertura de recolección de residuos sólidos no valorizables \\
\hline & & ¿Existe recolección de residuos sólidos valorizables? \\
\hline & & Producción per capita de residuos sólidos (Kg/Persona/día) \\
\hline & & $\begin{array}{l}\text { Inversión anual en Gestión Integral de Residuos Sólidos (GIRS) por persona } \\
\text { por año en el cantón (\$/persona/año) }\end{array}$ \\
\hline & & $\begin{array}{c}\text { Inversión en la limpieza de vias y áreas comunes por persona en cada } \\
\text { cantón (\$/persona/año) }\end{array}$ \\
\hline \multirow{13}{*}{$\begin{array}{c}\text { Gestión } \\
\text { Recurso } \\
\text { Hídrico }\end{array}$} & \multirow{7}{*}{$\begin{array}{l}\text { Gestión Protección } \\
\text { Recurso Hídrico }\end{array}$} & $\begin{array}{c}\text { ¿La ubicación de la captación está en área protegida o en zona de } \\
\text { conservación? }\end{array}$ \\
\hline & & ¿Está demarcada la zona de protección legalmente? \\
\hline & & ¿Se cuenta con información del balance hídrico? \\
\hline & & $\begin{array}{l}\text { Consumo promedio de agua de la comunidad expresado en unidades de } \\
\text { litros por persona por día (I/persona/día) }\end{array}$ \\
\hline & & ¿La ASADA posee plan de atención integral de riesgos? \\
\hline & & ¿Cuentan con programas para adaptación al cambio climático? \\
\hline & & ¿Posee registros de aforos de las fuentes de abastecimiento? \\
\hline & \multirow{4}{*}{$\begin{array}{l}\text { Calidad Agua } \\
\text { Potable }\end{array}$} & $\begin{array}{c}\text { ¿Se diagnóstica el riesgo en los componentes del acueducto mediante la } \\
\text { herramienta SERSA? }\end{array}$ \\
\hline & & ¿La ASADA posee sistema de desinfección? \\
\hline & & ¿Posee planta potabilizadora? \\
\hline & & ¿Posee la ASADA sello de calidad sanitaria? \\
\hline & \multirow{2}{*}{$\begin{array}{c}\text { Gestión } \\
\text { Administrativo / } \\
\text { Financiero }\end{array}$} & $\%$ de morosidad en el pago del servicio de agua \\
\hline & & Porcentaje (\%) de agua no contabilizada \\
\hline $\begin{array}{l}\text { Desarrollo } \\
\text { Social }\end{array}$ & $\begin{array}{l}\text { Índice de } \\
\text { Desarrollo Social }\end{array}$ & Índice de Desarrollo Social (IDS) en MIDEPLAN \\
\hline \multirow{2}{*}{ Educación } & \multirow{2}{*}{ Capacitaciones } & $\begin{array}{l}\text { ¿Posee distinción de bandera azul ecológica (en la comunidad, } \\
\text { microcuencas y municipalidades)? }\end{array}$ \\
\hline & & $\begin{array}{l}\text { ¿Los operadores han desarrollado programas de educación ambiental } \\
\text { para la comunidad y los imparten regularmente (una vez al año)? Si / No }\end{array}$ \\
\hline \multirow{4}{*}{$\begin{array}{l}\text { Riesgo por } \\
\text { Eventos } \\
\text { Naturales }\end{array}$} & \multirow{4}{*}{ Eventos Naturales } & ¿Posee su acueducto riesgo de inundación? \\
\hline & & ¿Posee su acueducto riesgo de deslizamientos? \\
\hline & & ¿Posee su acueducto riesgos volcánicos? \\
\hline & & ¿Su acueducto está ubicado zonas sísmicas? \\
\hline
\end{tabular}


La figura 5 muestra las fichas de metadatos que se construyeron para cada uno de los indicadores.

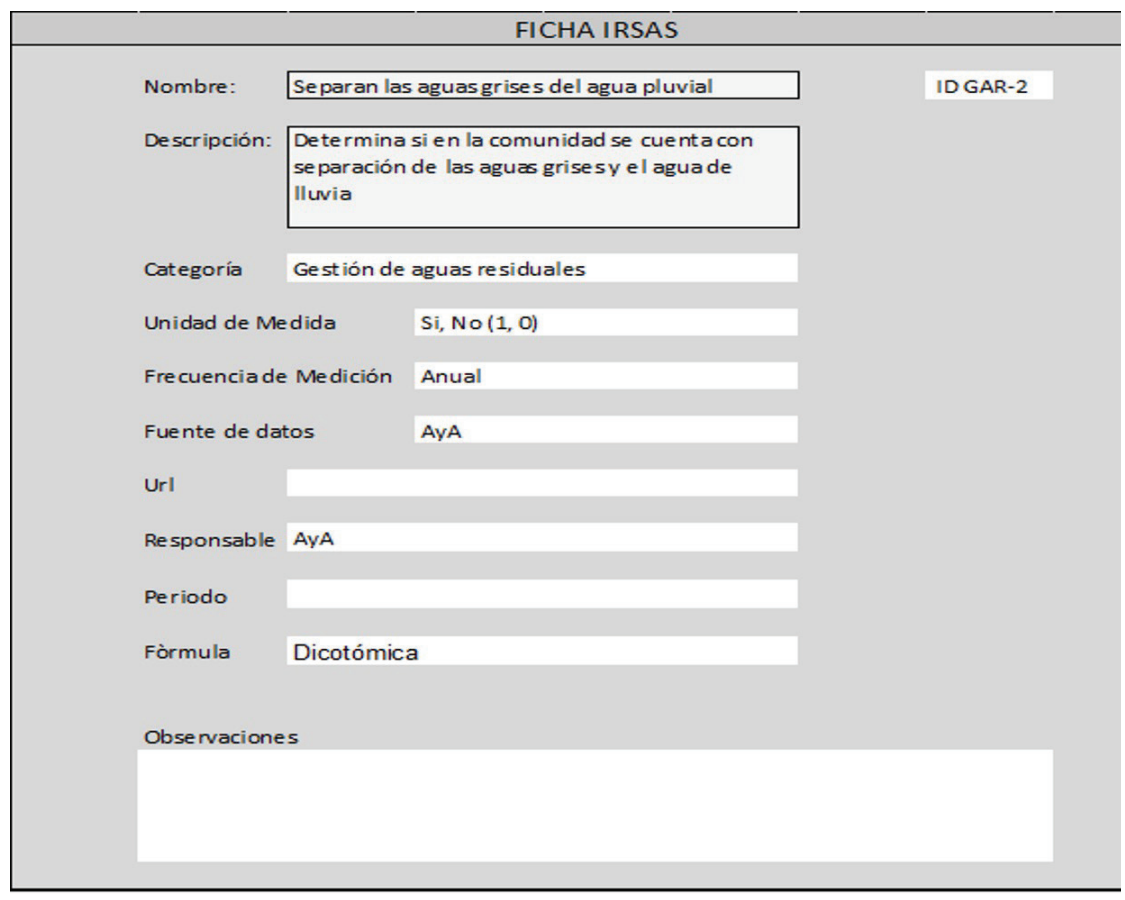

Figura 5. Ejemplo de ficha por indicador.

Adicionalmente, se acordó realizar la medición de los indicadores anualmente para obtener un IRSSAS por distrito.

\section{Identificación del riesgo en las ASADAs seleccionadas}

Con los resultados de la ecuación 1 aplicados a la muestra de estudio de 97 ASADAs, y mediante análisis estadísticos y visitas de campo, se definieron los ámbitos de clasificación del riesgo que se muestran en el cuadro 4, estas clasificaciones se asignaron geográficamente por distrito. Adicionalmente, los investigadores con base en las corroboración de la información en el campo, definieron el significado para cada nivel de riesgo, de esta forma los encargados de las ASADAs, funcionarios públicos de instituciones rectoras, público en general, pueden obtener retroalimentación útil, concisa para mejorar su gestión o informarse.

La unidad geográfica es el distrito, la forma que se representa el índice calculado, es asignándole un color según lo indicado en el cuadro 4.

A continuación, se muestra un ejemplo del mapa generado al aplicar el cálculo para obtener los IRSSAS (figura 6) por distrito, en el mismo se puede observar el riesgo asociado, cada ASADA está georreferenciada y se muestra como una gota de color azul. 
Cuadro 4. Descripción de la categoría del riesgo asociado al IRSSAS.

\begin{tabular}{|c|c|c|c|}
\hline $\begin{array}{c}\text { Clasificación de } \\
\text { riesgo }\end{array}$ & IRSSAS \% & Color & Significado \\
\hline Muy bajo & $<47$ & Azul & $\begin{array}{l}\text { La gestión del servicio de agua y del saneamiento es } \\
\text { sostenible. Es necesario para mantener el valor del } \\
\text { indicador, que se continúen implementando mejoras } \\
\text { para disminuir aún más el riesgo. }\end{array}$ \\
\hline Bajo & [47-57] & Celeste & $\begin{array}{l}\text { La vigilancia estricta en el sistema debe aplicarse con } \\
\text { el fin de mantener la sostenibilidad en la prestación } \\
\text { del servicio de agua y saneamiento. Es importante } \\
\text { implementar mejoras en los subcomponentes que } \\
\text { sobrepasen el } 47 \% \text {. }\end{array}$ \\
\hline Medio & [58-67] & Verde & $\begin{array}{c}\text { La gestión de la prestación del servicio de agua y } \\
\text { saneamiento es regular. Debe prestarse especial } \\
\text { atención a los subcomponentes con mayor porcentaje } \\
\text { de riesgo e implementar medidas correctivas a la } \\
\text { brevedad. }\end{array}$ \\
\hline Alto & [68-77] & & $\begin{array}{c}\text { El riesgo alto muestra una fragilidad del sistema servicio } \\
\text { de agua y saneamiento, es muy importante que se } \\
\text { revisen con detalle cada componente y subcomponente } \\
\text { del sistema para llevar a cabo las medidas correctivas } \\
\text { de forma inmediata de manera tal que se reduzca el } \\
\text { riesgo. }\end{array}$ \\
\hline Muy alto & $>77$ & & $\begin{array}{c}\text { La prestación del servicio de agua y saneamiento es } \\
\text { insostenible en el tiempo. Presenta un grave riesgo de } \\
\text { no poder brindarlo en un corto plazo. Se deben revisar } \\
\text { cada uno de los componentes y subcomponentes, para } \\
\text { tomar las medidas necesarias urgentes y reducir el } \\
\text { riesgo. }\end{array}$ \\
\hline
\end{tabular}
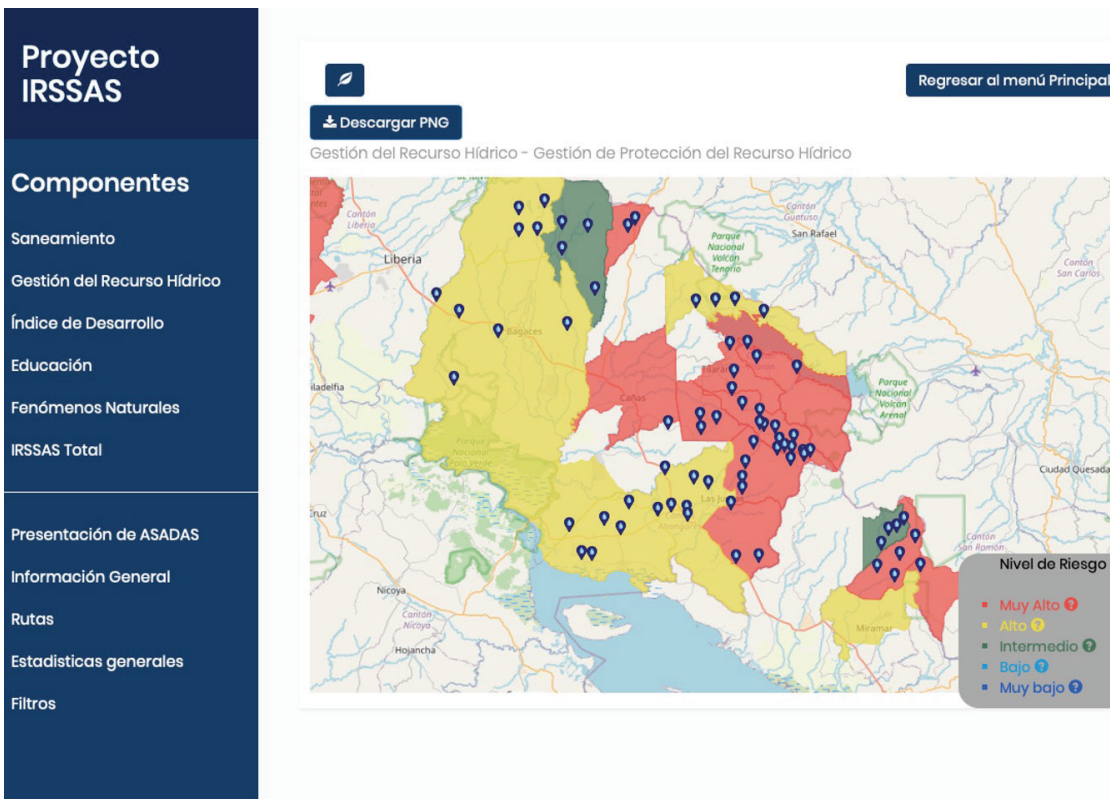

Gestión del Recurso Hidrico - Gestión de Protección del Recurso Hídrico

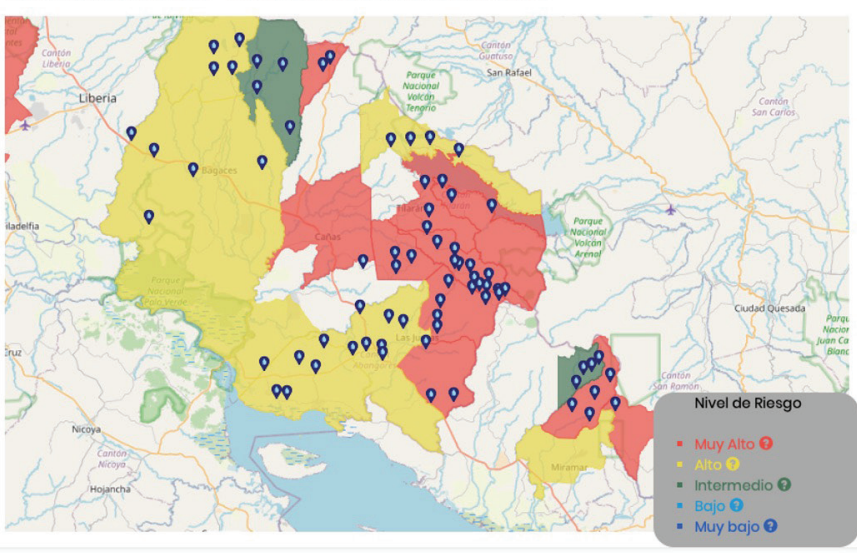

Figura 6. Fotografía del mapa generado por el software que calcula el IRSSAS. 
Los resultados del IRSSAS para la muestra de 97 ASADAs, se muestran en el cuadro 5 .

Cuadro 5. Clasificación del riesgo en la muestra seleccionada.

\begin{tabular}{|c|c|c|}
\hline Clasificación riesgo según IRSSAS & Cantidad de ASADAs & Porcentaje\% \\
\hline Muy alto & 4 & 4 \\
\hline Alto & 30 & 31 \\
\hline Intermedio & 45 & 46 \\
\hline Bajo & 15 & 15 \\
\hline Muy bajo & 3 & 3 \\
\hline TOTALES & 97 & 100 \\
\hline
\end{tabular}

Fuente: Resultados numéricos generados por la aplicación de la ecuación 1. Muestra de 97 ASADAs ubicadas en Guanacaste, Puntarenas y Cartago.

\section{Discusión y conclusiones}

El modelo de administración de agua mediante ASADAs presenta graves vulnerabilidades y es insostenible en el tiempo. Las amenazas sobre las ASADAs ya habían sido evidenciadas desde el 2004 [15], a la fecha aún se mantienen y se han profundizado con los años, como se mostró en el cuadro 2.

Los investigadores analizaron las vulnerabilidades y amenazas que mayormente inciden en la prestación del servicio y saneamiento y las clasificaron en cinco grandes bloques, denominados componentes.

El primero es el saneamiento ambiental, las ASADAs que podrán continuar prestando sus servicios en el tiempo, deben poner especial énfasis en controlar aquellas variables que afecten la calidad del agua, dentro de las cuales está la contaminación por aguas servidas, residuales, provenientes de actividades agroindustriales y residuos sólidos.

El segundo componente que afecta la prestación del servicio de agua y saneamiento es la gestión del recurso hídrico, y muy especialmente la gestión del agua potable en el acueducto (calidad, continuidad, cantidad) y la gestión administrativa y financiera (recursos para su funcionamiento e inversión en mejoras). Algunas de las fuentes que abastecen los acueductos están en manos privadas. Resulta de vital importancia garantizar la protección de las fuentes, con delimitación física para el acceso de personas y animales. Los acueductos rurales en el país capturan el agua desde manantiales, pozos y agua superficiales provenientes de la parte alta de la cuenca, la calidad del agua generalmente es muy buena, razón por la cual, el mayor riesgo se presenta en la capacidad de las ASADAs para evitar la contaminación en la fuente y el tratamiento de desinfección que realicen antes de su distribución.

Las ASADAs que no tienen la capacidad técnica ni tampoco los recursos para garantizar el proceso eficiente de desinfección presentan altos riesgos. Esto se suma a las dificultades inherentes de la escases del agua. Las ASADAs muy pequeñas, que administran menos de 300 abonados (76 \% de las ASADAs del país), facturan en promedio montos inferiores a 1,200,000 [4], un equivalente a 2100 dólares mensuales, con lo cual es imposible contar con disponibilidad financiera para invertir en operación mucho menos en mejoras de los acueductos.

Ante este panorama, es comprensible que las ASADAs no logren contratar sus propios estudios hidrogeologicos, los que existen a la fecha han sido el resultado de contrataciones directas por medio de AyA [12], igual situación ocurre con los planes de riesgo, educación ambiental y 
adaptación al cambio climático. Las ASADAs en el país sobreviven el día a día, experimentando grandes riesgos asociados a situaciones de emergencia como desastres naturales, o alguna epidemia, como es el caso del COVID19, donde se ha observado una importante disminución de ingresos por aumento de morosidad. Es imporante indicar que la existencia de muchas ASADAs, ha sido posible, gracias a las donaciones por parte del gobierno o ayudas externas, con las cuales se construyó el acueducto, sin embargo, muchas ASADAs, principalmente las más pequeñas, no son autosostenibles en el tiempo, por lo cual el riesgo en la prestación del servicio es muy alto.

El tercer componente que debe considerarse para garantizar la sostenibilidad de las ASADAs, es la capacidad de los habitantes para contribuir con el desarrollo del acueducto, por esta razón, los investigadores incorporaron el indicador desarrollado por MIDEPLAN [41] [38], que es compuesto, e integra factores socioeconómicos.

La educación ambiental está ligada con el uso sostenible del recurso, la conciencia para el manejo adecuado de los residuos sólidos y la responsabilidad social de evitar contaminación por aguas servidas, por tal razón, se incorporó este componente dentro de los aspectos a evaluar para la construcción del índice.

Finalmente, el quinto componente del IRSSAS, se relaciona con los eventos naturales, como inundaciones, deslizamientos, fallas sísmicas y volcanes, aspectos incontrolables pero que deben tenerse planes de contingencia ante tales.

En Costa Rica existen muchas estadísticas ambientales, que brindan una visión general del país, también se disponen de índices de primera y segunda generación de carácter descriptivo de la situación ambiental. Para lograr la sustentabilidad es necesario diseñar índices de tercera generación.

La construcción de un índice de tercera generación permite la planificación entre diversos sectores socio productivos. Esta investigación ha priorizado el sector de agua y saneamiento, debido a su elevada vulnerabilidad ante un escenario del cambio climático y el aumento de población.

EI IRSSAS está concebido como un indicador sistémico que combina la dimensión físicabiológica, social, económica y política, fue construido utilizando la lógica usada en la construcción de la huella ecológica [78], las guías metodológicas en la construcción de indicadores [55], la construcción de indicadores del uso de agua [35] y aplicando los parámetros usados por el MIDEPLAN para el manejo del territorio.

Su función es monitorear la calidad sin necesidad de realizar continuamente análisis físicoquímicos, continuidad y cantidad del agua potable para las poblaciones rurales, además, garantizar el mantenimiento de un ecosistema saludable para poder ofrecer disponibilidad de agua para la población actual y futura.

La distribución de agua potable y el saneamiento en Costa Rica presenta serios riesgos a mediano y largo plazo, debido a las diferentes debilidades del sector. La falta de herramientas de planificación orientadas a priorizar la inversión en infraestructura de recolección-distribución de agua potable, tratamiento de aguas negras residuos sólidos, protección de las fuentes de agua, prevención ante el cambio climático y medidas de mitigación ante las condiciones de vulnerabilidad asociadas al territorio.

Del análisis de los resultados obtenidos mediante la aplicación del índice IRSSAS se evidencia una situación negativa relacionada con la sustentabilidad en la prestación del servicio de agua y saneamiento, pues el 92 \% de las ASADAs se clasifican en riesgos de intermedio hacia arriba. 
Ante el cambio de los patrones de lluvia, que aumentan los desastres naturales y la oferta del agua, sumando al aumento de población, resulta necesario enfocar los esfuerzos en apoyar a los prestadores de agua en las zonas rurales, ya que los mismos presentan amenazas y vulnerabilidades multifactoriales, estás no han sido subsanadas en las últimas décadas.

La aplicación de este indicador evidencia nuevamente el riesgo inminente y puede servir como herramienta de planificación para priorizar inversiones y apoyar efectivamente a las ASADAs.

\section{Referencias}

[1] Acueductos y Alcantarillados, «La Gaceta, N 150, viernes 5 de agosto 2012. Reglamento de las Asociaciones Administradoras de Sistemas de Acueductos y Alcantarillados Comunales,» 2005. [En línea]. Available: https://www.aya.go.cr/ASADAS/Leyes\%20y\%20reglamentos/REGLAMENTO\%20DE\%20ASADAS.pdf.

[2] Gobierno de Costa Rica, Ley 2726, San José: La Gaceta, 1961.

[3] ARESEP, «Cobertura del servicio de acueducto por operador, zona y tipo de tubería 2017,» 2017. [En línea]. Available: https://aresep.go.cr/agua-potable/index.php?option=com_content\&view=article\&id=2471\&c atid=106.

[4] Acueductos y Alcantarillados, «Diagnostico Nacional de Entes Operadores de Agua en Zonas Rurales,» 2020. [En línea]. Available: https://www.aya.go.cr/ASADAS/Documents/Diagnóstico\%20Nacional\%20de\%20 Entes\%20Operadores\%20(Levantamiento\%202017-2020).pdf.

[5] A. Navarro-Garro, F. Araya-Rodriguez, D. Pérez-Murillo, C. Moreira-Segura y M. Estrada-Ugalde, «Vulnerabilidad de los sistemas de acueductos rurales: cómo identificarla,» Tecnología en marcha, vol. 26, n 3, pp. 63-74, 2013.

[6] M. González-Pérez y F. Jimenez, «Gestión del agua para consumo humano de las asociaciones administradoras de acueductos y alcantarillados (ASADAS) de Buenos Aires y de Suerre, Área de conservación Tortuguero, Costa Rica, Aqua-Lac, vol. 5, n 1, pp. 1-21, 2013.

[7] Repositorio Universidad Nacional de Costa Rica, «Elaboración de un plan de seguridad del agua para la ASADA Rincón de Zaragoza,» 2018. [En línea]. Available: https://repositorio.una.ac.cr/bitstream/handle/11056/15115/TFG_Ana\%20Gabriela\%20Chac\%c3\%b3n\%20Herrera.pdf?sequence=1\&isAllowed=y.

[8] Repositorio Universidad Nacional de Costa Rica, «Herramienta para la mejora de la gestión físico-sanitaria de 10 Asadas de la región Pacífico Central de Costa Rica,» 2017. [En línea]. Available: https://repositorio.una.ac.cr/ bitstream/handle/11056/13659/PROYECTO\%20DE\%20GRADUACI\%c3\%93N.pdf?sequence=1\&isAllowed=y.

[9] A. Suárez-Serrano, A. Baldioceda-Garro, G. Durán-Sanabria, J. Rojas-Conejo, D. Rojas-Cantillano y A. GuillénWatson, «Seguridad hídrida: Gestión del agua en comunidades rurales del Pacífico Norte de Costa Rica,» Revista de Ciencias Ambientales. Tropical Journal of Environmental Sciences, vol. 53, nº 2, 2019.

[10] Repositorio Institucional TEC, «Evaluación general del sistema de agua potable y aspectos básicos de saneamiento de la ASADA Agrimaga, ubicada en el cantón de Guácimo, en Limón, influenciado por el Acuífero Guácimo-Pococí,» 2018. [En línea]. Available: https://repositoriotec.tec.ac.cr/bitstream/ handle/2238/10092/evaluacion_general_sistema_agua_potable_aspectos_basicos_saneamiento_asada. pdf? sequence $=1 \&$ is Allowed $=y$.

[11] Repositorio Universidad Nacional, «Plan de seguridad del agua de la ASADA las Vueltas, Parrita, Puntarenas, » 2019. [En línea]. Available: http://repositorio.ucr.ac.cr/bitstream/handle/10669/80372/TFG\%2c\%20Natalia\%20 Garcia\%20Campos. . pdf?sequence=1\&isAllowed=y.

[12] Repositorio institucional de Acueductos y Alcantarillados, «Estudio hidrogeológico para la determinación de la zona de protección absoluta y zona de recarga de las nacientes de Desamparaditos, Puriscal, Costa Rica,» 2018. [En línea]. Available: http://dspace-aya.eastus.cloudapp.azure.com:8080/xmlui/handle/aya/164.

[13] Repositorio Institucional Acueductos y Alcantarillados, «Estudio hidrogeológico, Determinación de la zona de protección de la naciente Asada San Martín de León Cortes.,» 2015. [En línea]. Available: http://dspace-aya.eastus.cloudapp.azure.com:8080/xmlui/bitstream/handle/aya/185/CEDO\%20AyA\%20AD\%205200. pdf?sequence $=1$.

[14] Kerwa, Universidad de Costa Rica, «Análisis de vulnerabilidad ambiental por deslizamiento en la microcuenca del río Tarbacia, canton de Mora, República de Costa Rica,» 2019. [En línea]. Available: http://www.kerwa.ucr. ac.cr/bitstream/handle/10669/80292/Tesis_Carbajal_Vald\%c3\%a9s.pdf?sequence=1\&isAllowed=y. 
[15] Repositorio institucional AyA, «Estudio de Aspectos Institucionales de desarrollo de los acueductos Rurales en Costa Rica, Lockwood, H,» 2004. [En línea]. Available: http://dspace-aya.eastus.cloudapp.azure.com:8080/ xmlui/bitstream/handle/aya/335/CEDO\%20AyA\%201900. pdf?sequence=1\&isAllowed=y.

[16] Repositorio Acueductos y Alcantarillados, «Lo intangible de los planes de seguridad del agua,» 2011. [En línea]. Available: https://www.aya.go.cr/centroDocumetacion/catalogoGeneral/Lo\%20intangible\%20de\%20 los\%20planes\%20de\%20seguridad\%20del\%20agua.pdf.

[17] C. R. Diagnóstico técnico y administrativo de cinco ASADAs localizadas dentro del corredor biologico Garcimuñiz. Provincia Alajuela, «Repositorio Universidad Tecnica Nacional, UTN,» 2016. [En línea]. Available: http://repositorio.utn.ac.cr/handle/123456789/128.

[18] Repositorio Universidad Técnica Nacional, (UTN), «IV Encuentro Nacional de Asociatividad en la Gestión Comunitaria del Agua,» 2019. [En línea]. Available: http://repositorio.utn.ac.cr/handle/123456789/356.

[19] Instituto Metereologico Nacional, «Mejoramiento de las capacidades nacionales para la evaluación de la vulnerabilidad y adaptación del sistema hídrico al cambio climático en Costa Rica, como mecanismo para disminuir el riesgo al cambio climático y aumental el índice de desarrollo humano,» 2012. [En línea]. Available: http://cglobal.imn.ac.cr/documentos/publicaciones/AdaReHiCRCC/offline/download.pdf.

[20] Climate Expert ORG, «Riesgo futuro del sector hídrico ante el cambio climático,» 2012. [En línea]. Available: https://www.climate-expert.org/fileadmin/user_upload/PDF/Costa_Rica/riesgo_futuro.pdf.

[21] Repositorio Universidad Estatal a Distancia (UNED), «Plan de Acción de la estrategia Nacional de Cambio Climático,» 2009. [En línea]. Available: https://www.uned.ac.cr/extension/images/ifcmdl/amas/recursos/cambio-climatico/plan-de-accion-estrategia-nacional-cambio-climatico.pdf.

[22] Gobierno de Costa Rica, «Política Nacional de adaptación al cambio climático de Costa,» 2018. [En línea]. Available: http://www.pgrweb.go.cr/DocsDescargar/Normas/No\%20DE-41091/Nersion1/Politica_ ADAPTACION_24_abril.pdf.

[23] Ministerio de Ambiente y Energía, «Plan Nacional de Descarbonización,» 2018. [En línea]. Available: http:// www.pgrweb.go.cr/DocsDescargar/Normas/No\%20DE-41091/Version1/Politica_ADAPTACION_24_abril.pdf.

[24] Instituto Nacional de Estadísticas y Censos (INEC), «Indicadores ambientales INEC,» 2010-2016. [En línea]. Available: https://www.inec.cr/medio-ambiente/indicadores-ambientales.

[25] Universidad de Costa Rica, "Costa Rica salda una deuda pendiente: indicadores ambientales ya son de acceso público,» 2019. [En línea]. Available: https://www.ucr.ac.cr/noticias/2019/07/04/costa-rica-salda-unadeuda-pendiente-indicadores-ambientales-ya-son-de-acceso-publico.html.

[26] Ministerio de Ambiente y Energía, «Sistema de Consulta de indicadores para el SINIA,» [En línea]. Available: http://sinia.go.cr/estadistica/. [Último acceso: 2020].

[27] Dirección de Aguas del Ministerio de Ambiente y Energía (MINAE), «Estadísticas de agua Costa Rica,» 2019. [En línea]. Available: http://www.da.go.cr/estadisticas-e-indicadores-del-agua/.

[28] CEPAL, «Avance de estadísticas ambientales en Costa Rica,» 2020. [En línea]. Available: https://www.cepal. org/sites/default/files/courses/files/2_2-avance-ea-costa-rica_minae.pdf.

[29] CONARE, «Programa Estado de la Nación,» Sistema Universitario Estatal de Costa Rica, 2020. [En línea]. Available: https://estadonacion.or.cr.

[30] Gobierno de Costa Rica, «Estado del Ambiente en Costa Rica,» 2018. [En línea]. Available: https://www.presidencia.go.cr/comunicados/2018/02/costa-rica-presenta-el-primer-informe-oficial-del-estado-del-amnbiente/.

[31] Banco Central de Costa Rica, «Sistema de Contabilidad Ambiental,» 2019. [En línea]. Available: https://www. bccr.fi.cr/seccion-cuentas-ambientales/cuentas-ambientales.

[32] Ministerio de Salud, «Costa Rica recibe aval de la OCDE en materia ambiental,» 2019. [En línea]. Available: https://www.ministeriodesalud.go.cr/index.php/noticias/noticias-2019/1465-costa-rica-recibe-aval-de-la-ocdeen-materia-ambiental.

[33] K. Lezzaik, A. Milewski y J. Mullen, «The groundwater risk index: Deveolpment and applicaction in the Middle East and North Africa Region," Science of the Total Environment, Vols. \%1 de \%2628-629, pp. 1149-1164, 2018.

[34] B. R, A survey of composite indeces measuring country perfomance 2008, New York: United Nations Development Programe, 2008.

[35] S. Sandoval-Solis y C. McKinney, «Sustainabilty Index for Water Resources Planning and Management,» Journal of Water Resources Planning and Management, vol. 137, n 5, 2011. 
[36] J. Ganoulis, «Integrated Risk Analysis for sustainable water resources management,» de Comparative Risk Assessment and Environmental Decision Making. Nato Science Series: IV Earth and Enviromental Sciences, vol. 38, Springer, Dordrech, 2004, pp. 275-286.

[37] A. Sarang, A. Vahedi y A. Shamsai, «How to Quantify Sustainable Development: A Risk-Based Approach to Water Quality Management,» Environmental Management, vol. 41, pp. 200-220, 2008.

[38] Ministerio de Planificación, Costa Rica, «Indice de desarrollo social,» 2018. [En línea]. Available: https://www. mideplan.go.cr/indice-desarrollo-social.

[39] Contraloría General de la República, Costa Rica, «Sistema Integrado de Información Municipal,» 2018, [En línea]. Available: https://cgrweb.cgr.go.cr/apex/t?p=142:8:0:

[40] Construcción de indicadores ambientales, «Repositorio de la CEPAL,» 2014. [En línea]. Available: https:// repositorio.cepal.org/bitstream/handle/11362/5502/1/S0900307_es.pdf.

[41] Ministerio de Planificación MIDEPLAN, «Indice de Desarrollo Social,» 2020. [En línea]. Available: https://www. mideplan.go.cr/indice-desarrollo-social.

[42] Ministerio de Salud, «Indicadores del Ministerio de Salud,» 2020, [En línea]. Available: https://www.ministeriodesalud.go.cr/index.php/acceso-a-sistemas-de-informacion.

[43] Instituto Nacional de Fomento Municipal, «Capacitaciones,» 2020, [En línea]. Available: http://www.ifam. go.cr/?page_id=1689.

[44] Contraloría General de la República, «Indice de Gestión Municipal,» 2019, [En línea]. Available: https://cgrfiles.cgr.go.cr/publico/docsweb/documentos/publicaciones-cgr/igm/2018/igm-2018.pdf.

[45] D. Mora, V. Mata y C. Portuguez, «Agua para consumo y saneamiento, situación de Costa Rica en el contexto de las Américas, 1961-2011,» Mayo 2012. [En línea]. Available: http://www.paho.org/cor/index. php?option=com_docman\&view=download\&category_slug=salud-y-ambiente\&alias=219-agua-para-consumo-y-saneamiento-situacion-de-costa-rica-en-el-contexto-de-las-americas-1960-2011\&ltemid=222.

[46] Comisión Nacional de prevención del riesgo y atención de Emergencias CNE, «Mapas de Amenazas,» 2020. [En línea]. Available: https://www.cne.go.cr/reduccion_riesgo/mapas_amenzas/index.aspx.

[47] Instituto Nacional de Estadísticas y Censos, Costa Rica, «Resultados de la Encuesta Nacional de Hogares,» Julio 2016. [En línea]. Available: http://sistemas.inec.cr/pad4/index.php/catalog/165/datafile/F2/V588.

[48] A. Gain, C. Giupponi y Y. Wada, «Measuring global water security towards sustainable development goals," Enviromental research letters, vol. 11, $\mathrm{n}^{\circ}$ 12, 2016.

[49] Instituto Geográfico Nacional, «Sistema Nacional Infraestructura Nacional de Datos Espaciales de Costa Rica,» Gobierno de Costa Rica, 2020. [En línea]. Available: http://www.snitcr.go.cr/instituto_geografico_nacional.

[50] S. Soto-Córdoba, L. Gavira-Montoya y M. Pino-Gomez, «Estudio de caso: disposición de las aguas residuales domésticas en zonas rurales de Costa Rica,» Ambiente e Sociedade, vol. 22, pp. 1-20, 2019.

[51] S. Soto-Córdoba, L. Gaviria-Montoya y M. Pino-Gomez, «Situación de la gestión del agua potable en las zonas rurales de la provincia de Cartago, Costa Rica,» Tecnología en Marcha, pp. 67-76, 2016.

[52] Reglamento para la calidad del agua N 38924-S, "Sistema Costarricense de información jurídica," 2015. [En línea]. Available: http://www.pgrweb.go.cr/scij/Busqueda/Normativa/Normas/nrm_texto_completo. aspx?nValor1=1\&nValor2=80047.

[53] Formulario Unificado AyA, «Acueductos y Alcantarillados,» 2017. [En línea]. Available: https://www.aya.go.cr/ ASADAS/documentacionAsadas/FORMULARIO\%20UNIFICADO.pdf.

[54] Metodologías para evaluar la amenaza, «Repositorio Gestión del Riesgo, Colombia,» 2018. [En línea]. Available: https://repositorio.gestiondelriesgo.gov.co/bitstream/handle/20.500.11762/27226/Metodologias_ evaluar_amenaza_ciclones\%20tropicales. pdf?sequence=4\&isAllowed=y.

[55] A. Schuschny y H. Soto, "Guía metodológica diseño de indicadores compuestos de desarrollo sostenible,» Naciones Unidas, Santiago de Chile, 2009.

[56] «Metodología Delphi,» 2020. [En línea]. Available: https://research.phoenix.edu/content/research-methodology-group/delphi-method) .

[57] Repositorio ITCR, «Evaluación de la gestión de la ASADA COOPEROSALES, San Nicolás, Cartago, desde una perspectiva del uso sostenible del recurso hídrico.,» 2016. [En línea]. Available: https://repositoriotec.tec. ac.cr/handle/2238/7040. 
[58] Repositorio TEC, «Evaluación de la gestión de la ASADA de Quebradilla, Quebradilla, Cartago, desde una perspectiva del uso sostenible del recurso hídrico,» 2016. [En línea]. Available: https://repositoriotec.tec.ac.cr/ handle/2238/7016.

[59] Repositorio TEC, «Evaluación de la gestión de la ASADA de Guatuso, San Isidro, El Guarco, desde una perspectiva del uso sostenible del recurso hídrico,» 2016. [En línea]. Available: https://repositoriotec.tec.ac.cr/ handle/2238/7015.

[60] Repositorio TEC, «Evaluación de la gestión de la ASADA de Higuito, San Isidro, El Guarco, desde una perspectiva del uso sostenible del recurso hídrico,» 2016. [En línea]. Available: https://repositoriotec.tec.ac.cr/ handle/2238/7017.

[61] Repositorio Institucional TEC, «Evaluación de la gestión de la ASADA de Guatuso, San Isidro, El Guarco, desde una perspectiva del uso sostenible del recurso hídrico,» [En línea]. Available: https://repositoriotec.tec. ac.cr/handle/2238/7015.

[62] Repositorio ITCR, «Evaluación de la gestión de la ASADA de Cañón-Macho Gaff Damita, San Isidro, El Guarco, desde una perspectiva del uso sostenible del recurso hídrico,» [En línea]. Available: https://repositoriotec.tec. ac.cr/handle/2238/7014.

[63] Repositorio ITCR, «Evaluación de la gestión de la ASADA de Cot, Cot, Oreamuno, desde una perspectiva del uso sostenible del recurso hídrico,» 2016. [En línea]. Available: https://repositoriotec.tec.ac.cr/handle/2238/7011.

[64] Repositorio TEC, «Evaluación de la gestión de la ASADA de Paso Ancho y Boqueron, Cipreses, Oreamuno, desde una perspectiva del uso sostenible del recurso hídrico ,» 2016. [En línea]. Available: https://repositoriotec.tec.ac.cr/handle/2238/7010.

[65] Repositorio TEC, «Evaluación de la gestión de la ASADA de Potrero Cerrado, Potrero Cerrado, Oreamuno, desde una perspectiva del uso sostenible del recurso hídrico,» 2016. [En línea]. Available: https://repositoriotec.tec.ac.cr/handle/2238/7009.

[66] Repositorio TEC, «Evaluación de la gestión de la ASADA de San Pablo, Santa Rosa, Oreamuno, desde una perspectiva del uso sostenible del recurso hídrico,» 2016. [En línea]. Available: https://repositoriotec.tec.ac.cr/ handle/2238/7006.

[67] Repositorio TEC, «Evaluación de la gestión de la ASADA de Santa Rosa, Santa Rosa, Oreamuno, desde una perspectiva del uso sostenible del recurso hídrico,» 2016. [En línea]. Available: https://repositoriotec.tec.ac.cr/ handle/2238/7008.

[68] Repositorio ITCR, «Evaluación de la gestión de la ASADA de El Yas, Santiago, Paraíso, desde una perspectiva del uso sostenible del recurso hídrico,» 2016. [En línea]. Available: https://repositoriotec.tec.ac.cr/handle/2238/7007.

[69] Repositorio del ITCR, «Evaluación de la gestión de la ASADA del Palomo, Orosí, Paraíso, desde una perspectiva del uso sostenible del recurso hídrico,» 2016. [En línea]. Available: https://repositoriotec.tec.ac.cr/ handle/2238/7005.

[70] Repositorio TEC, «Evaluación de la gestión de la ASADA de Río Macho, Orosi, Paraíso, desde una perspectiva del uso sostenible del recurso hídrico,» 2016. [En línea]. Available: https://repositoriotec.tec.ac.cr/handle/2238/7003.

[71] Repositorio TEC, «Evaluación de la gestión de la ASADA San Jerónimo, Cachí, Paraíso, desde una perspectiva del uso sostenible del recurso hídrico,» 2016. [En línea]. Available: https://repositoriotec.tec.ac.cr/handle/2238/7002.

[72] Repositorio TEC, «EVALUACIÓN DE LA GESTIÓN DE LA ASADA DE SANTIAGO, SANTIAGO, PARAÍSO, DESDE UNA PERSPECTIVA DEL USO SOSTENIBLE DEL RECURSO HÍDRICO,» 2017. [En línea]. Available: https:// repositoriotec.tec.ac.cr/bitstream/handle/2238/7001/17\%20santiago_revisado.pdf?sequence=1\&isAllowed=y.

[73] Repositorio TEC, «Evaluación de la gestión de la ASADA de San Vicente, San Rafael, La Unión, desde una perspectiva del uso sostenible del recurso hídrico, , 2016. [En línea]. Available: https://repositoriotec.tec. ac.cr/handle/2238/7012.

[74] Repositorio ITCR, «Evaluación de la gestión de la ASADA de Alto Varas, La Isabel, Turrialba, desde una perspectiva del uso sostenible del recurso hídrico,» 2016. [En línea]. Available: https://repositoriotec.tec.ac.cr/ handle/2238/7024.

[75] Repositorio TEC, «Evaluación de la gestión de la ASADA de Jicotea, Tayutic, Turrialba, desde una perspectiva del uso sostenible del recurso hídrico,» 2016. [En línea]. Available: https://repositoriotec.tec.ac.cr/handle/2238/7004. 
[76] Repositorio TEC, «Evaluación de la gestión de la ASADA de Santa Cristina, La Suiza, Turrialba, desde una perspectiva del uso sostenible del recurso hídrico,» 2016. [En línea]. Available: https://repositoriotec.tec.ac.cr/ handle/2238/7000.

[77] Repositorio TEC, «Evaluación de la gestión de la ASADA de Santa Cruz, Santa Cruz, Turrialba, desde una perspectiva del uso sostenible del recurso hídrico ,» 2016. [En línea]. Available: https://repositoriotec.tec. ac.cr/handle/2238/7023.

[78] M. Wackernagel y W. Rees, «Nuestra huella ecológica: Reduciendo el impacto humano sobre la Tierra,,» Polis, Revista Latinoamericana, 2003. 